\title{
Odontogenic myxofibroma- Report of a rare entity
}

\author{
Poudel $\mathrm{P}^{1}$, Bajracharya $\mathrm{B}^{1}$, Bhattacharyya $\mathrm{S}^{1}$, Bajracharya $\mathrm{D}^{1}$, Singh $\mathrm{S}^{2}$, \\ Pokhrel $\mathrm{P}^{2}$, Desar B ${ }^{2}$ \\ ${ }^{1}$ Department of Oral and Maxillofacial Pathology, Kantipur Dental College, Kathmandu, Nepal \\ ${ }^{2}$ Department of Oral and Maxillofacial Surgery, Kantipur Dental College, Kathmandu, Nepal
}

\section{Keywords: \\ Fibromyxoma; Myxofibroma; Myxoma; \\ Odontogenic}

\begin{abstract}
Odontogenic myxofibroma is a rare, benign tumor, which is considered to be the variant of odontogenic myxoma. It is locally infiltrative, aggressive and has high recurrence rate. Only 24 specific cases of myxofibroma have been reported since 1950 and only two cases have been reported with excessive calcifications till 2012. Here, we report a rare case of Odontogenic myxofibroma with calcifications in 53 years old female patient who presented with the chief complain of swelling. The final diagnosis of Odontogenic myxofibroma was established after histopathological and immunohistochemical examination.
\end{abstract}

\section{INTRODUCTION}

Odontogenic myxofibroma is a rare, benign and locally aggressive tumor first described by Virchow in $1863 .{ }^{1}$ They are considered as the variant of odontogenic myxoma. ${ }^{2} \mathrm{Of}$ all odontogenic tumor, 3-6\% are represented by myxomas and myxofibromas comprise small number of all myxomas., ${ }^{2,3}$ Initially the lesion may be small and asymptomatic but later on large lesions may cause painless expansion of buccal and lingual cortical plates. ${ }^{2}$ Radiographically, the lesion presents as unilocular or multilocular radiolucent area with well defined or diffuse margin. Histologically, these tumors consist of loose abundant mucoid ground substance with little collagen fibers. The amount of this collagen determines whether they are called myxofibromas or myxomas. ${ }^{3}$ Herein we report a case of myxofibroma of mandible in a 53 years old female patient along with its immunohistochemical analysis.

\section{Correspondence:}

Dr. Dipshikha Bajracharya

Assistant Professor

Department of Oral and Maxillofacial Pathology

Kantipur Dental College, Basundhara, Kathmandu

Email:drdipshikhabaj@gmail.com

\section{CASE REPORT}

Fifty three years old female patient reported with chief complain of swelling on the right side of lower jaw since six months. On clinical examination, hard swelling was seen on buccal aspect of tooth w.r.t 43, 44, 45 and 46. 44, 45 were missing and 46, 47,48 were mobile. The overlying mucosa was ulcerated.

On radiographic examination, multilocular radiolucency extending from the region of 41 to the right ramus of the mandible was seen. (fig.1) CBCT finding revealed a solitary multilocular lytic hypoattenuated area extending from apical aspect of 41 to the right ramus of the mandible. The margin of the lesion was thin, well defined, sclerotic and scalloped. There was thinning, expansion and perforation of buccal and lingual cortical plate of bone. Internal structure of lesion was homogenous with presence of thin septas. The surrounding bone had normal trabecular pattern and there was no evidence of root resorption of the adjacent teeth. On the basis of clinical and radiographical findings, 


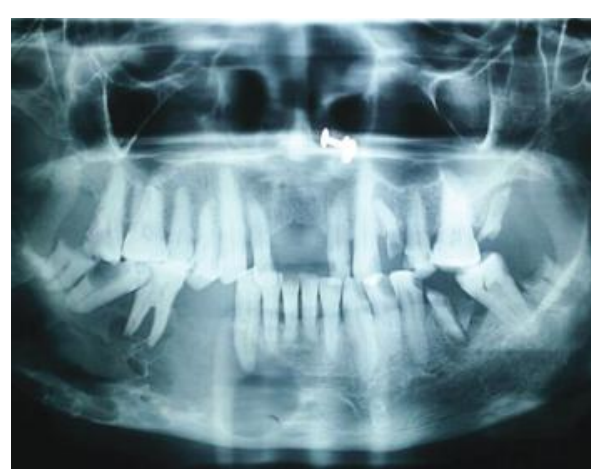

Figure 1: OPG showing multilocular radiolucency extending from midline to right ramus of mandible.

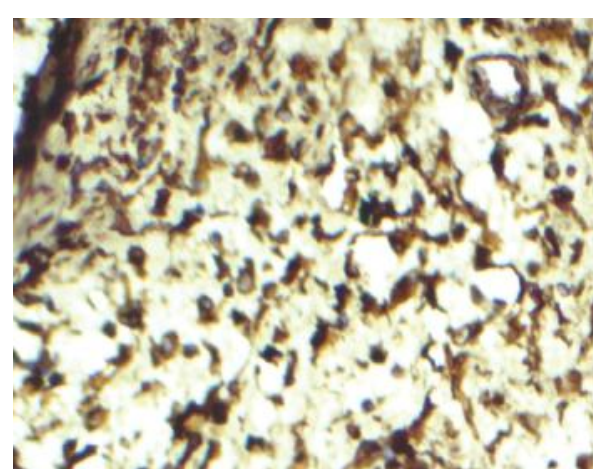

Figure 3: Photomicrograph showing diffusely positive vimentin marker (IHC stain,X40).

diagnosis of $\mathrm{OKC}$ in the right body and ramus was given. The incisional biopsy of the lesion was taken and sent for histopathological examination.

On gross examination, the tissue was smooth, whitish in color, firm in consistency and gritty at certain areas. Microscopic examination of the tissue revealed tumor mass composed of myxomatous stroma with abundant loosely arranged collagen fibers. Tumor cells were composed of stellate and spindle shaped fibroblasts. Remnants of odontogenic epithelium and areas of cementum like calcifications were also noted at places. Based on these findings histopathological diagnosis of odontogenic fibromyxoma was given. Segmental resection and reconstruction was done as treatment and resected mandible was sent for histopathological examination.

Resected mandible specimen of about $8.7 \mathrm{~cm} \times 3.5 \mathrm{~cm} \times 2.2$ $\mathrm{cm}$ in size was received. Soft tissue from both buccal and lingual side was excised. Grossly the tissue was glistening, white with smooth surface and firm in consistency. On microscopic examination, myxomatous stroma with loosely arranged collagen fibers were seen. Remnants of nonactive odontogenic epithelium and few areas of cementum like calcification were also seen in the stroma. (fig.2) Thus the final diagnosis of odontogenic myxofibroma

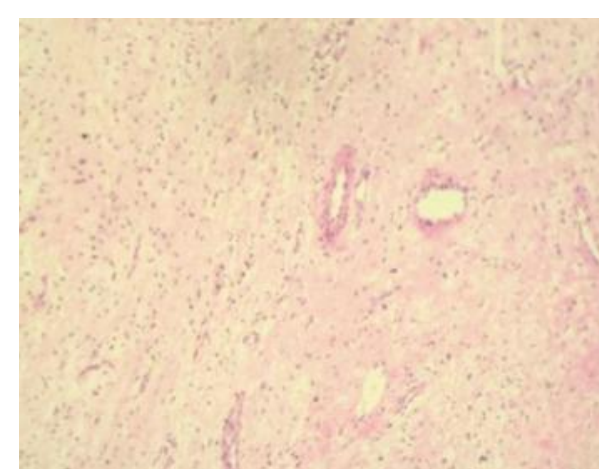

Figure 2: Photomicrograph showing myxomatous connective tissue with collagen fibers (HE stain,x 100).

was given. To confirm the histopathological diagnosis, immunohistochemistry (IHC) was done using peroxidase. The result was diffusely positive for vimentin, (fig.3) positive for SMA, negative for S-100. Ki-67 was less than $1 \%$. IHC finding suggested low grade myxomatous lesion. Hence the final diagnosis of odontogenic myxofibroma was confirmed.

\section{DISCUSSION}

Odontogenic myxofibroma is a rare benign, locally aggressive odontogenic tumor that can cause extensive bony destruction and infiltration into the surrounding structure. ${ }^{4,5}$ The origin of this tumor is considered to be the mesenchyme of the developing tooth or periodontal ligament. Some authors have considered this lesion to be the myxomatous change of odontogenic fibroma or residual foci of embryonic tissue. There is still some debate regarding the origin of this tumor. ${ }^{6}$ However its frequent occurrence in tooth bearing part of jaw, histological similarity to dental papilla and dental follicle, association with unerupted or missing teeth, presence of odontogenic epithelium in some cases and rarity of this tumor in extragnathic bone supports the odontogenic origin of this tumor. ${ }^{7}$

The incidence of this tumor is found to be 0.05 new cases per million population per year. It most frequently occurs between the age of 10 and 30 years. Females are affected more commonly than males and mostly affects posterior mandible. ${ }^{2}$ Smaller lesions do not present any symptoms loosened teeth may draw attention large lesion are often associated with cortical plate expansion and perforation. ${ }^{2}$

Radiographically, the lesion presents as unilocular or multilocular radiolucency exhibiting soap-bubble or honeycomb or tennis racquet appearance with a well-defined or diffuse margin. Occasionally, there may be expansion of buccal and lingual cortical plates ${ }^{7}$ which was seen in our case.

Histologically, odontogenic myxofibromas are characterized by the presence of spindle and stellate shaped cells in the 
myxoid stroma. There is presence of prominent collagen fibers which differentiates this lesion from myxoma. ${ }^{3}$ Occasionally, remnants of odontogenic epithelium are present in the stroma. In our case the presence of spindle and stellate shaped cells in myxoid stroma along with collagen fibers were important in establishing the diagnosis. In addition to these features our case showed few calcified products through out the lesion which is similar to the finding of Sriram et al. ${ }^{4}$ The neoplastic spindle cells are fibroblast like cells called myxoblasts which secrete large amount of mucopolysaccharides. The ground substance consist of $80 \%$ hyaluronic acid and $20 \%$ chondroitin sulphate. The excessive amount of hyaluronic acid is responsible for local invasiveness of this neoplasm. ${ }^{1}$

On immunohistochemical analysis, Vimentin was found to be diffusely positive, smooth muscle actin was positive, $\mathrm{S}-100$ was negative and $\mathrm{Ki}-67$ was $<1 \%$. These findings suggested the low grade myxoid lesion and based on all these findings final diagnosis of myxofibroma was confirmed.

The treatment of choice for odontogenic myxofibroma is surgery. This involves enucleation and curettage, radical surgery and en block resection depending on the size of tumor. ${ }^{8,6}$ There have been higher recurrence rate for this tumor between $25 \%$ and $43 \% .^{8}$ This recurrence is mostly due to incomplete removal of tumor rather than true recurrence. ${ }^{7}$

\section{CONCLUSION}

Odontogenic myxofibroma is benign but locally aggressive neoplasm of the jaw. If excision or curettage is incomplete, it tends to recur. Hence long term surveillance and regular follow should be maintained irrespective of the treatment modality.

\section{REFERENCES}

1. Rani V, Kadar MKM, Babu A, Sankari L, Krishnasamy G. Odontogenic myxoma diagnostic dilemma : A case report and review of literature. $\mathrm{J}$ Cranio-Maxillary Dis. 2014;3:163-7.

2. Meleti M, Giovannacci I, Corradi D, Manfredi M, Merigo E. Odontogenic myxofibroma: A concise review of the literature with emphasis on the surgical approach. Med Oral Patol Oral Cir Bucal. 2015;20:1-6.

3. Burak A, Ali M, Bilgic B. Myxofibroma of the maxilla , current concepts, and differential diagnosis. J Dent Sci. 2013;4-7.

4. Sriram K, Lavanya S, Ramesh V, Premalatha B. Odontogenic myxofibroma with abundant calcifications, masquerading as a fibroosseous lesion: A diagnostic dilemma. Indian J Oral Sci. 2012;3:99.

5. Infante-cossío $\mathrm{P}$, Martínez-de-fuentes $\mathrm{R}$, García-perla-garcía A, Jiménez-caste- E. Myxofibroma of the maxilla . Reconstruction with iliac crest graft and dental implants after tumor resection. Med Oral Patol Oral Cir Bucal. 2011;16:532-6.

6. Simon ENM, Merkx MAW, Vuhahula E, Ngassapa D. Odontogenic myxoma : a clinicopathological study of 33 cases. Int J Oral Maxillofac Surg. 2004;33:333-7.

7. Reichart PA, Philipsen HP. Odontogenic tumors and allied lesions. Quintessence Pub.; 2004.p.77-86.

8. Reddy G, Kumar BS, Muppa R, Regonda SK, TVS HK. Odontogenic fibromyxoma of maxilla: a rare case report. Case Rep Dent. 2013;345479. doi: 10.1155/2013/345479 